\title{
Do Arabic numerals activate magnitude automatically? Evidence from the psychological refractory period paradigm
}

\author{
Natalie Ford $^{1}$ • Michael G. Reynolds ${ }^{1}$
}

Published online: 29 March 2016

(C) Psychonomic Society, Inc. 2016

\begin{abstract}
A single experiment $(N=30)$ tested the claim that Arabic numerals automatically activate their magnitude representations by assessing whether central attention is required in order to activate magnitude within the psychological refractory period (PRP) paradigm. Subjects performed a color discrimination task as Task 1 and a parity judgment task as Task 2 . Task overlap was controlled by varying stimulus onset asynchrony (SOA). A spatial-numerical association of response codes (SNARC) effect arose in Task 2 and yielded under-additive effects with decreasing SOA. This result suggests that magnitude is activated prior to central attention, and is therefore consistent with the claim that numerals activate magnitude automatically.
\end{abstract}

Keywords Attention - Mathematical cognition ·

Psychological refractory period paradigm $\cdot$ Automaticity

The claim that Arabic numerals (e.g., 4) activate their magnitude representations automatically is pervasive in the numerical cognition literature (e.g., Cohen Kadosh, Henik, \& Rubinsten, 2008; Dehaene, Bossini, \& Giraux, 1993; Fischer, Castel, Dodd, \& Pratt, 2003; Gevers, Lammertyn, Notebaert, Verguts, \& Fias, 2006; Mapelli, Rusconi, \& Umiltà, 2003; Schwarz \& Keus, 2004). Many investigators have tested this claim by demonstrating that magnitude is activated unintentionally in tasks where activating magnitude is unnecessary for correct task performance, such as naming (e.g., Brysbaert, 1995), parity judgment (e.g., Dehaene et al.,

Michael G. Reynolds

michaelchanreynolds@trentu.ca

1 Department of Psychology, Trent University, Peterborough, Ontario, Canada K9J 7B8
1993), and physical size comparison (e.g., Cohen Kadosh et al., 2008).

However, assessing whether magnitude is activated unintentionally is not an exhaustive test of automaticity, as many other characteristics have been attributed to automatic processes (e.g., LaBerge \& Samuels, 1974; Pashler, 1994; Treisman, Vieira, \& Hayes, 1992). For instance, automatic processes are also argued to not require processing capacity (e.g., Pashler, 1994), to proceed in parallel with other processes (e.g., Treisman et al., 1992), and to not use attention (LaBerge \& Samuels, 1974). Thus, many of the characteristics that have been attributed to automatic processes amount to these processes not requiring attentional resources (e.g., Logan, 1988).

Assessing whether a process requires attention is complicated by the fact that research has distinguished between at least two separate attentional systems: (1) input attention, which arises at an early stage of processing and is involved in the parallel perceptual processing of multiple stimuli, and (2) central attention, which arises at a later stage of processing and is involved in response selection (Johnston, McCann, \& Remington, 1995; Lavie, Hirst, de Fockert, \& Viding, 2004; Pashler, 1994; Reimer, Strobach, Frensch, \& Schubert, 2015; Wickens, 2002). These dissociable attentional systems have their own capacity limits (e.g., Lavie et al., 2004; Wickens, 2002) and involve different cognitive mechanisms (Johnston et al., 1995; Reimer et al., 2015). Critically, assessing whether a process requires input attention assesses whether it requires domain-specific perceptual resources, whereas assessing whether a process requires central attention assesses whether it requires more general resources involved in response selection (Johnston et al., 1995; Pashler, 1994; Lavie et al., 2004; Wickens, 2002).

Consistent with numerals not requiring input attention in order to activate magnitude, Blanc-Goldhammer and Cohen 
(2014) and Pashler and Badgio (1987) found no benefit to comparing the magnitude of four numerals that were presented successively as opposed to simultaneously within Shiffrin and Gardener's (1972) time delimitation paradigm. Thus, multiple numerals can activate magnitude in parallel. The goal of the present experiment was to assess whether activating magnitude requires central attention.

\section{The present experiment}

The psychological refractory period (PRP) paradigm has been used to examine whether processes use central attention. Within this paradigm, subjects perform two speeded tasks (Task 1 and Task 2) with priority given to Task 1 . Task overlap is controlled by varying stimulus onset asynchrony (SOA). At short SOAs (e.g., $50 \mathrm{~ms}$ ), processing for the two tasks largely overlaps, whereas at long SOAs (e.g., 2,000 ms), processing for the two tasks does not overlap at all. The standard finding is that Task 2 response time (RT) increases as SOA decreases. The most widely used interpretation of this finding is that both tasks use the same limited capacity processor, which acts as an all-or-none central processing bottleneck involved in response selection (e.g., Pashler, 1994). However, the bottleneck may affect processes as early as stimulus categorization (Johnston \& McCann, 2006). According to the all-or-none bottleneck account, Task 2 slowing with decreasing SOA occurs because Task 2 central processing is delayed until the processor is freed by Task 1 central processing. In contrast, Task 2 processes that occur before or after the bottleneck do not use the limited capacity processor (or central attention) and can therefore proceed in parallel with Task 1 central processes. ${ }^{1}$

Insight into whether a process uses central attention can be gained by examining how the effects of a Task 2 factor are affected by increasing task overlap. According to locus of slack logic (e.g., Pashler \& Johnston, 1989), the delay of Task 2 central processing at short SOAs creates a period of cognitive slack during which Task 2 central processing waits for the processing bottleneck to be freed by Task 1 (see Fig. 1, panel a). This period of cognitive slack can absorb the effects of a Task 2 factor that affects processing prior to the central processing bottleneck. Thus, the effects of a pre-bottleneck factor (i.e., one that does not require central attention) will be reduced or eliminated at short SOAs. However, if the effects of a Task 2 factor arise at or after the bottleneck (and therefore require central attention), the effects of this factor will arise after the period of cognitive slack and consequently be of the same magnitude across SOAs (see Fig. 1, panels b and c). Because locus of slack logic clearly predicts how a

\footnotetext{
${ }^{1}$ For the present purposes, bottleneck theories that postulate capacity sharing between the two tasks (e.g., Tombu \& Jolicœur 2003) make the same predictions as the all-or-none bottleneck account.
}

factor that uses central attention will behave when two tasks overlap, the PRP paradigm has been used to investigate whether processes arise automatically (e.g., McCann, Remington, \& Van Selst, 2000; Reynolds \& Besner, 2006).

The present experiment tested the claim that numerals automatically activate magnitude by investigating whether the spatial-numerical association of response codes (SNARC) effect (Dehaene et al., 1993) arises in the absence of central attention. The SNARC effect refers to the finding that lefthand key presses are executed faster than right-hand key presses in response to small numbers, whereas right-hand key presses are executed faster than left-hand key presses in response to large numbers. The SNARC effect has been attributed to the activation of an internal, analogue number line with small numbers located on the left side and large numbers located on the right side (Dehaene et al., 1993; Gevers et al., 2006; Mapelli et al., 2003; Schwarz \& Keus, 2004; but see van Dijck \& Fias, 2011). This effect has been observed in parity judgment (e.g., Dehaene et al., 1993), magnitude comparison (e.g., Gevers, Verguts, Reynvoet, Caessens, \& Fias, 2006), and phoneme detection (Fias, Brysbaert, Geypens, \& d'Y dewalle, 1996). We elected to use the SNARC effect here because it (1) is thought to reflect the activation of number magnitude and (2) arises in the absence of intentional strategies (e.g., Dehaene et al., 1993; see Tzelgov \& Ganor-Stern, 2005). ${ }^{2}$

In the present experiment, subjects performed a color discrimination task as Task 1 and a parity judgment task as Task 2. Trials were labelled "congruent" when subjects executed left-hand responses in response to small numbers (1-4) and right-hand responses in response to large numbers (6-9), and "incongruent" when subjects executed left-hand responses in response to large numbers and right-hand responses in response to small numbers. The SNARC effect was derived by calculating the difference in RT on congruent and incongruent trials. The size of this effect was compared across long and short SOAs. If the SNARC effect decreases as SOA decreases, this would be consistent with numerals activating magnitude in the absence of central attention, or automatically, whereas if the SNARC effect is additive with SOA, this would be consistent with numerals requiring central attention in order to activate magnitude, and therefore not activating magnitude automatically.

\footnotetext{
${ }^{2}$ Several previous studies have examined how Arabic numerals are affected by SOA in the PRP paradigm; however, the implications of these studies for automaticity are unclear. For instance, both additive and underadditive effects of magnitude and SOA have been reported using the magnitude comparison task (Sigman and Dehaene, 2005, 2006). Further, Muller and Schwarz (2007) reported that the SNARC effect was additive with SOA in the parity judgment task; however, their long SOA condition was only $400 \mathrm{~ms}$, which means that Task 2 central processing was likely delayed by Task 1 central processing at long SOAs. In light of these considerations, further examination of how numerals activate magnitude using the PRP paradigm is necessary.
} 


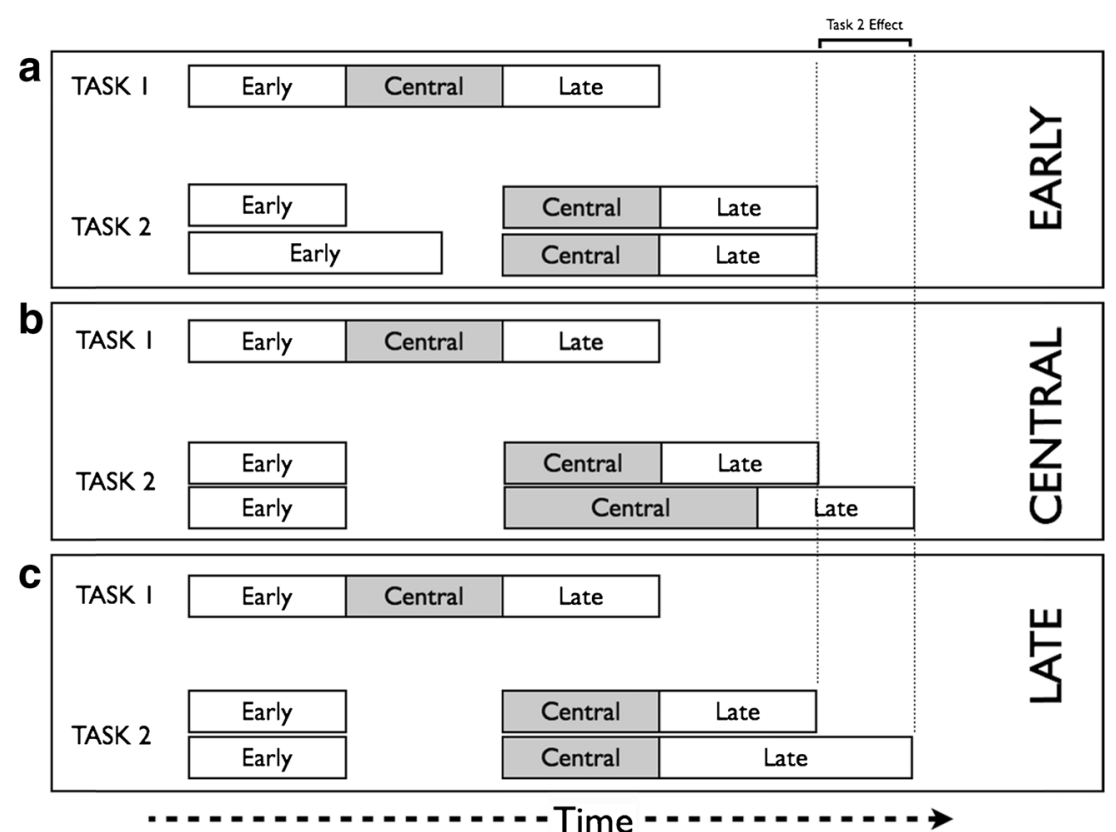

Fig. 1 An illustration of locus of slack logic in the context of the psychological refractory period (PRP) paradigm. Panel a illustrates absorption of an early effect into cognitive slack. Panels $\mathbf{b}$ and $\mathbf{c}$ illustrate how central and late effects in Task 2 are unaffected by task overlap

\section{Method}

Participants Thirty undergraduate Trent University students participated in the experiment in return for credit in an eligible psychology course. All participants had normal or correctedto-normal vision.

Stimuli The stimulus for Task 1 was a rectangle that subtended $1.6^{\circ}$ by $1.6^{\circ}$ visual angle. The border of this rectangle was three pixels thick and alternated unpredictably across trials between red and blue. The stimuli for Task 2 were Arabic numerals ranging in value from $0-9$. Numerals were presented in a white 18-pt. Courier New font against a black background and subtended $.6^{\circ}$ by $.6^{\circ}$ visual angle.

Apparatus The experiment was conducted on a Dell Vostro 420 computer running Microsoft Windows XP operating system. Stimulus presentation and data collection were controlled using E-Prime 2.0 software. Task 1 vocal responses were collected using a PST response box and voice-key assembly. Task 2 manual responses were collected using a standard USB keyboard.

Procedure The experiment consisted of two blocks, each with 48 practice trials and 216 experimental trials. Subjects were given the opportunity to take a break between blocks. Subjects responded to Task 1 by saying the color into a microphone. Subjects responded to Task 2 by indicating parity with key presses (" $\mathrm{X}$ " and " $\mathrm{M}$ "). The assignment of key to parity changed halfway through the experiment, and the order of key-to-parity assignment was counterbalanced pseudorandomly across subjects based on the order in which they were tested.

At the beginning of each trial, a rectangle appeared in the center of the screen. The border of the rectangle was initially white and changed to either red or blue after $500 \mathrm{~ms}$. A numeral then appeared in the center of the rectangle after an SOA of 50,150 , or $2,000 \mathrm{~ms}$. When a vocal response was registered, the border of the rectangle returned to white. In order to encourage subjects to give priority to Task 1 , they received feedback on trials where Task 1 RTs were longer than 1,500 $\mathrm{ms}$, they responded to Task 2 before Task 1, or an interval of less than $100 \mathrm{~ms}$ separated the two responses. Feedback remained on the screen for 3,500 ms. On trials where feedback was not given, a blank feedback screen was presented for 100 ms. Accuracy of the vocal response (correct, incorrect, or a voice-key failure) was coded via key press by the researcher after the feedback screen, but before the beginning of the next trial.

Subjects were instructed to respond to both tasks as quickly and accurately as possible, but with priority given to Task 1 . They were seated approximately $50 \mathrm{~cm}$ from the computer screen. The experiment took approximately $45 \mathrm{~min}$ to complete.

\section{Results}

Separate robust repeated measures ANOVAs were run on the $20 \%$ Winsorized means (Wilcox, 1998) of the RT and error 
data for Task 1 and Task 2 with SOA (50 ms vs. 150 ms vs. 2, $000 \mathrm{~ms}$ ) and congruency (congruent vs. incongruent) as factors. Yuen's (1974) t-tests were used to examine simple effects. Robust statistical methods were used because they address issues of non-normality and heteroscedasticity (Wilcox, 1998).

Trials where the numerals 0 or 5 were presented were excluded from the analyses. The numeral 0 was excluded because responses to this item are typically error-prone and highly variable (e.g., Brysbaert, 1995; Nuerk, Iversen, \& Klaus, 2004). The numeral 5 was excluded because congruency between magnitude and response side was contingent on whether the numeral was greater than or less than 5 .

\section{Response time (RT)}

Prior to analyzing the RT data, trials were removed where an error was made in Task $1(0.510 \%)$ or Task $2(3.64 \%)$ or a voice-key failure occurred in Task $1(1.35 \%)$. Mean RTs for both tasks can be seen in Fig. 2, panel a.

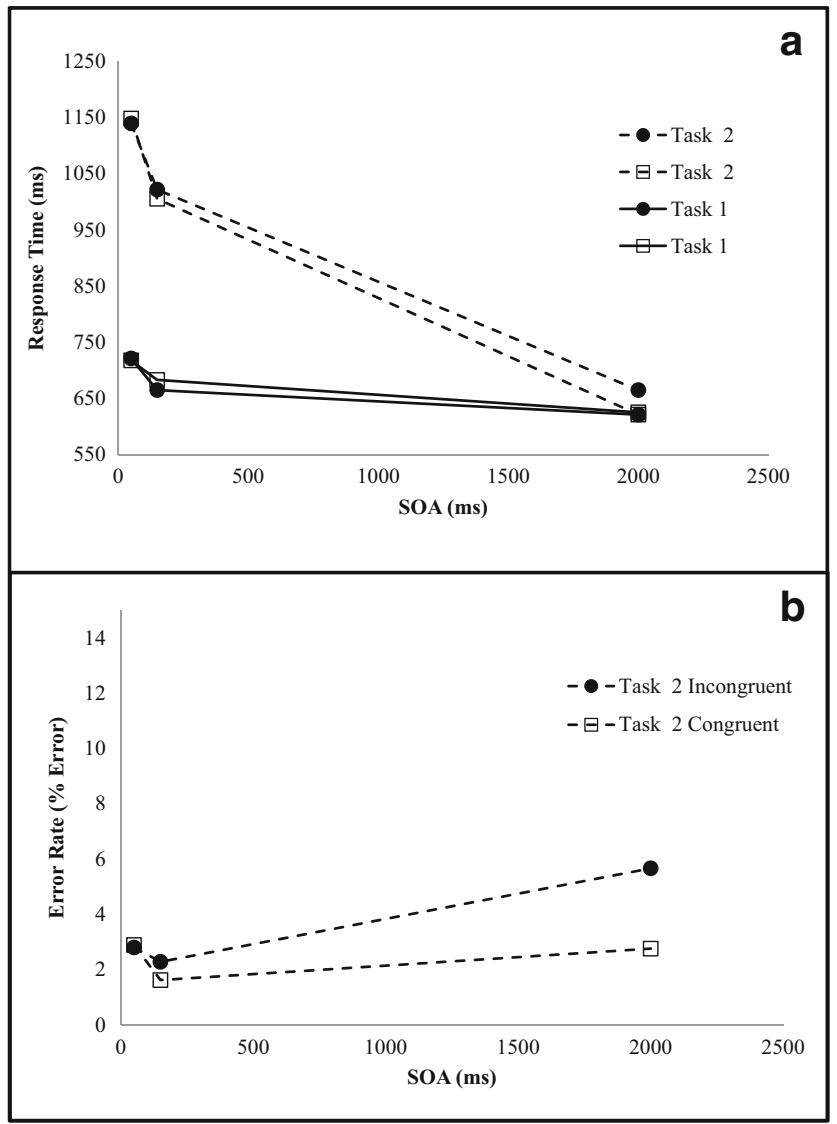

Fig. 2 Response time (panel a) and percentage error (panel b) for color discrimination (Task 1) and parity judgment (Task 2) tasks as a function of stimulus onset asynchrony (SOA) and congruency between magnitude and response side (spatial-numerical association of response codes (SNARC) effect)
Task 1: Color discrimination The time taken to name colors increased as SOA decreased, $F=16.34, p<$ .001. Critically, color naming times did not differ as a function of Task 2 congruency, $F=0.015, p=.902$, and the interaction between SOA and congruency did not approach significance, $F=1.28, p=.279$. These results are consistent with subjects giving priority to Task 1 .

Task 2: Parity judgment The time taken to make parity judgments increased as SOA decreased, $F=544.85, p<$ .001. Overall, there was a 15-ms SNARC effect, $F=8.07$, $p=.004$. Consistent with numerals activating magnitude in the absence of central attention, SOA and congruency interacted, whereby the SNARC effect decreased in size with decreasing SOA, $F=7.79, p<.001$. There was a significant 40-ms SNARC effect at SOAs of 2,000 ms, $t=$ $4.89, p<.001$, a non-significant $11-\mathrm{ms}$ SNARC effect at SOAs of $150 \mathrm{~ms}, t=0.420, p=.680$, and a nonsignificant $-5-\mathrm{ms}$ SNARC effect at SOAs of $50 \mathrm{~ms}, t=$ $-0.165, p=.871$.

\section{Percentage error}

Task 1: Color discrimination Because there were too few errors $(<1 \%)$, the error data for Task 1 were not analyzed.

Task 2: Parity judgment The error data for Task 2 can be seen in Fig. 2, panel b. A SNARC effect was observed in the error data, whereby more errors were made on incongruent trials than on congruent trials, $F=4.14, p=.041$. More errors were also made on long SOA trials than on short SOA trials, $F=8.43, \mathrm{p}<.001$, but this result can be attributed to an interaction between SOA and congruency, whereby the SNARC effect decreased in size as SOA decreased, $F=4.12, p=.017$. There was a significant SNARC effect at SOAs of $2,000 \mathrm{~ms}, t=3.14, p<.01$, and non-significant SNARC effects at SOAs of 150 and 50 ms $(t=1.11, p=.281$, and $t=0.134, p=.895$, respectively).

\section{Discussion}

The goal of the present experiment was to test the claim that Arabic numerals activate their magnitude representations automatically. Prior research investigating this claim has demonstrated that magnitude is activated unintentionally (e.g., Dehaene et al., 1993) and in the absence of input attention (e.g., Blanc-Goldhammer \& Cohen, 2014). Here, automaticity was tested by assessing whether the SNARC effect requires central attention. Consistent with numerals activating magnitude automatically, the SNARC effect yielded under- 
additive effects with decreasing SOA within the PRP paradigm.

\section{Locus of the SNARC effect}

There has been some debate concerning the stage of processing at which the SNARC effect arises. Some investigators have argued that the SNARC effect arises at response selection because it (1) requires subjects to respond using a lateralized manual effector (e.g., Keus \& Schwarz, 2005) and (2) interacts with other effects that are thought to arise at response selection (e.g., Gevers, Caessens, \& Fias, 2005). Inconsistent with this account, the SNARC effect went under-additive with decreasing SOA in the present experiment, which suggests that the SNARC effect arises prior to response selection. The present data are therefore more consistent with the claim that the links between magnitude and space are activated early in processing (e.g., Schwarz \& Keus, 2004). Consistent with this account, evidence suggests that the magnitude of numerals can bias spatial (input) attention, as small numbers shift attention to the left whereas large numbers shift attention to the right (Fischer, 2001; Fischer et al., 2003).

\section{Working memory account}

So far, we have interpreted the SNARC effect as arising from the activation of spatially oriented magnitude representations held in long-term memory (e.g., Dehaene et al., 1993). However, recent evidence suggests that the SNARC effect may arise from temporary representations held in working memory (van Dijck \& Fias, 2011). Consistent with the SNARC effect arising in working memory, it is eliminated during parity judgments when there is a concurrent verbal working memory load (van Dijck et al., 2009). Further, when a classification task is performed on a list of items maintained in working memory, a SNARC-like effect arises that reflects ordinal position in working memory (i.e., faster left-hand responses are executed in response to items located early in the ordinal sequence and faster right-hand responses are executed in response to items located late in the ordinal sequence; Ginsburg et al., 2014; van Dijck \& Fias, 2011). Given evidence that central attention is involved in consolidating items into visual short-term memory (e.g., Brisson \& Jolicoeur, 2007; Marois \& Ivanoff, 2005), the present observation that the SNARC effect arose in the absence of central attention is inconsistent with the SNARC effect arising from temporary representations held in working memory. However, the present results would be consistent with the working memory account if it is assumed that working memory activates links between magnitude and space that are held in long-term memory (see Ginsburg et al., 2014 for a discussion of this account). The most straightforward explanation is that the temporary associations between magnitude and space established using working memory can be activated by incoming stimuli early in processing (i.e., prior to central attention). Further research into the relationship between central attention, working memory, and the SNARC effect is certainly required.

\section{Conclusion}

Numerous investigators have claimed that Arabic numerals activate their magnitude representations automatically. Consistent with this claim, previous research has demonstrated that numerals activate magnitude unintentionally (e.g., Dehaene et al., 1993) and in the absence of input attention (e.g., Blanc-Goldhammer \& Cohen, 2014). Here, we demonstrated that numerals do not require central attention in order to activate magnitude (e.g., Pashler, 1994). Thus, our results converge on the claim that numerals activate magnitude automatically.

Author Note This work was supported by grants 341586 to MGR from the Natural Sciences and Engineering Research Council of Canada.

\section{References}

Blanc-Goldhammer, D. R., \& Cohen, D. J. (2014). Unlimited capacity parallel quantity comparison of multiple integers. Journal of Experimental Psychology: Learning, Memory, and Cognition, 40, 1389-1403.

Brisson, B., \& Jolicoeur, P. (2007). A psychological refractory period in access to visual short-term memory and the deployment of visualspatial attention: Multitasking processing deficits revealed by eventrelated potentials. Psycholphysiology, 44, 323-333.

Brysbaert, M. (1995). Arabic number reading: On the nature of the numerical scale and the origin of phonological recoding. Journal of Experimental Psychology: General, 124, 434-452.

Cohen Kadosh, R., Henik, A., \& Rubinsten, O. (2008). Are Arabic and verbal numbers processed in different ways? Journal of Experimental Psychology: Learning, Memory, and Cognition, 34, 1377-1391.

Dehaene, S., Bossini, S., \& Giraux, P. (1993). The mental representation of parity and number magnitude. Journal of Experimental Psychology: General, 122, 371-396.

Fias, W., Brysbaert, M., Geypens, F., \& d'Ydewalle, G. (1996). The importance of magnitude information in numerical processing: Evidence from the SNARC effect. Mathematical Cognition, 2, 95-110.

Fischer, M. H. (2001). Number processing induces spatial performance biases. Neurobiology, 57, 822-826.

Fischer, M. H., Castel, A. D., Dodd, M. D., \& Pratt, J. (2003). Perceiving numbers causes spatial shifts of attention. Nature Neuroscience, 6 , 555-556.

Gevers, W., Caessens, B., \& Fias, W. (2005). Towards a common processing architecture underlying Simon and SNARC effects. European Journal of Cognitive Psychology, 17, 659-673.

Gevers, W., Lammertyn, J., Notebaert, W., Verguts, T., \& Fias, W. (2006). Automatic response activation of implicit spatial information: Evidence from the SNARC effect. Acta Psychologica, 122, 221233. 
Gevers, W., Verguts, T., Reynvoet, B., Caessens, B., \& Fias, W. (2006). Numbers and space: A computational model of the SNARC effect. Journal of Experimental Psychology: Human Perception and Performance, 32, 32-44.

Ginsburg, V., van Dijck, J. P., Previtali, P., Fias, W., \& Gevers, W. (2014). The impact of verbal working memory on number-space associations. Journal of Experimental Psychology: Learning, Memory, and Cognition, 40, 976-986.

Johnston, J. C., \& McCann, R. S. (2006). On the locus of dual-task interference: Is there a bottleneck at the stimulus classification stage? The Quarterly Journal of Experimental Psychology, 59, 694-719.

Johnston, J. C., McCann, R. S., \& Remington, R. W. (1995). Chronometric evidence for two types of attention. Psychological Science, 6, 365-369.

Keus, I. M., \& Schwarz, W. (2005). Searching for the functional locus of the SNARC effect: Evidence for a response-related origin. Memory \& Cognition, 33, 681-695.

LaBerge, D., \& Samuels, S. J. (1974). Toward a theory of automatic information processing in reading. Cognitive Psychology, 6, 293323.

Lavie, N., Hirst, A., de Fockert, J. W., \& Viding, E. (2004). Load theory of selective attention and cognitive control. Journal of Experimental Psychology: General, 133, 339-354.

Logan, G. D. (1988). Toward an instance theory of automatization. Psychological Review, 95, 492-527.

Mapelli, D., Rusconi, E., \& Umiltà, C. (2003). The SNARC effect: an instance of the Simon effect? Cognition, 88, B1-B10.

Marois, R., \& Ivanoff, J. (2005). Capacity limits of information processing in the brain. Trends in Cognitive Sciences, 9, 296-305.

McCann, R. S., Remington, R. W., \& Van Selst, M. (2000). A dual-task investigation of automaticity in visual word processing. Journal of Experimental Psychology: Human Perception and Performance, 26, 1352-1270.

Muller, D., \& Schwarz, W. (2007). Exploring the mental number line: evidene from a dual task paradigm. Psychological Research, 71, 798-613.

Nuerk, H. C., Iversen, W., \& Willmes, K. (2004). Notational modulation of the SNARC and the MARC (linguistic markedness of response codes) effect. Quarterly Journal of Experimental Psychology Section, 57A, 835-863.

Pashler, H. (1994). Dual-task interference in simple tasks: Data and theory. Psychological Bulletin, 116, 220-244.

Pashler, H., \& Badgio, P. C. (1987). Attentional issues in the identification of alphanumeric characters. In M. Coltheart (Ed.), Attention and
Performance XII: The Psychology of Reading (pp. 63-82). Hove, England: Erlbaum.

Pashler, H., \& Johnston, J. C. (1989). Chronometric evidence for central postponement in temporally overlapping tasks. The Quarterly Journal of Experimental Psychology, 41, 19-45.

Reimer, C. B., Strobach, T., Frensch, P. A., \& Schubert, T. (2015). Are processing limitations of visual attention and response selection subject to the same bottleneck in dual-tasks? Attention, Perception, \& Psychophysics, 77, 1052-1069.

Reynolds, M., \& Besner, D. (2006). Reading aloud is not automatic: Processing capacity is required to generate a phonological code from print. Journal of Experimental Psychology: Human Perception and Performance, 32, 1303-1323.

Schwarz, W., \& Keus, I. M. (2004). Moving the eyes along the mental number line: Comparing SNARC effects with saccadic and manual responses. Perception \& Psychophysics, 66, 651-664.

Sigman, M., \& Dehaene, S. (2005). Parsing a cognitive task: A characterization of the mind's bottleneck. PLoS Biology, 3, 334-349.

Sigman, M., \& Dehaene, S. (2006). Dynamics of the central bottleneck: Dual-task and task uncertainty. PLoS Biology, 4, 1227-1238.

Shiffrin, R. M., \& Gardner, G. T. (1972). Visual processing capacity and attentional control. Journal of Experimental Psychology, 93, 72-82.

Tombu, M., \& Jolicœur, P. (2003). A central capacity sharing model of dual-task performance. Journal of Experimental Psychology: Human Perception and Performance, 29, 3-18.

Treisman, A., Vieira, A., \& Hayes, A. (1992). Automaticity and preattentive processing. The American Journal of Psychology, 105, 341-362.

Tzelgov, J., \& Ganor-Stern, D. (2005). Automaticity in processing ordinal information. In J. I. D. Campbell (Ed.), Handbook of Mathematical Cognition (pp. 43-54). New York: Psychology Press.

van Dijck, J. P., \& Fias, W. (2011). A working memory account for spatial-numerical associations. Cognition, 119, 114-119.

van Dijck, J. P., Gevers, W., \& Fias, W. (2009). Numbers are associated with different types of spatial information depending on the task. Cognition, 113, 248-253.

Wickens, C. D. (2002). Multiple resources and performance predition. Theoretical Issues in Ergonomics Science, 3, 159-177.

Wilcox, R. R. (1998). The goals and strategies of robust methods. British Journal of Mathematical and Statistical Psychology, 51, 1-39.

Yuen, K. K. (1974). The two-sample trimmed $t$ for unequal population variances. Biometrika, 61, 165-170. 